\title{
Seroprevalencia de la enfermedad de Chagas en embarazadas del departamento de Cordillera en el período 2010-2016 y el comportamiento de la seroprevalencia después de 21 años de la implementación del Programa de Control Prenatal de Chagas
}

\author{
*Graciela Meza Acosta ${ }^{1}$, Hugo Cerecetto Meyer ${ }^{2}$ \\ ${ }^{1}$ Universidad Nacional de Asunción, Instituto de Investigaciones en Ciencias de la Salud. Paraguay \\ ${ }^{2}$ Universidad de la República, Uruguay, Facultad de Ciencias, Centro de Investigaciones Nucleares. Uruguay
}

Cómo referenciar este artículo/ How to reference this article:

\begin{abstract}
Meza Acosta G, Cerecetto Meyer H. Seroprevalencia de la enfermedad de Chagas en embarazadas del departamento de Cordillera en el período 2010-2016 y el comportamiento de la seroprevalencia después de 21 años de la implementación del Programa de Control Prenatal de Chagas. Mem. Inst. Investig. Cienc. Salud. 2019; 17(3): $10-19$
\end{abstract}

\section{RES U MEN}

En Paraguay se estima que 165.000 personas están infectadas por Trypanosoma cruzi, agente etiológico de la enfermedad de Chagas, de las cuales 61.000 son mujeres en edad fértil. En 1995 se implementó el Programa de Control Prenatal de Chagas en el departamento de Cordillera, zona endémica del país. El objetivo de este estudio es analizar el comportamiento de la seropositividad a $T$. cruzi en embarazadas en 21 años de control prenatal (período: 1995-2016), con énfasis en el periodo 2010-2016. El estudio, descriptivo de corte trasversal retrospectivo, empleó los datos obtenidos de los registros de la III Región Sanitaria y de los cinco laboratorios de diagnóstico. Se emplearon los resultados de la serología de 20 distritos en un total de 23.661 embarazadas que asistieron a su control prenatal. En los registros figuran 1.074 embarazadas seropositivas, distribuidas en los cinco laboratorios de referencia, resultando el $5 \%$ la prevalencia de infección con $T$. cruzi en el período 2010-2016. Con una prevalencia al inicio del período de $16 \%$, se evidenció el principal descenso a $6 \%$ en el 2014, situación que se mantuvo en una fase cuasi estacionaria hasta el 2016. Se concluye que el sistema de diagnóstico prenatal de la enfermedad de Chagas asociado a los métodos de prevención implementados por el Ministerio de Salud Pública y Bienestar Social, han resultado efectivos. Esta información es útil para, a través de la identificación de las embarazadas seropositivas y el tratamiento correspondiente, controlar la transmisión congénita.

Palabras clave: Embarazadas, control prenatal, enfermedad de Chagas, seroprevalencia.

Fecha de recepción: abril 2019. Fecha de aceptación: julio 2019

*Autor correspondiente: Graciela Meza Acosta. Universidad Nacional de Asunción, Instituto de Investigaciones en Ciencias de la Salud. Paraguay.

Email: graciemezaacosta@gmail.com 


\title{
Seroprevalence of Chagas disease in pregnant women of the department of Cordillera in the period 2010- 2016 and the behavior of the seroprevalence after 21 years of Prenatal Chagas Control Program implementation
}

\begin{abstract}
A B S T R A C T
It is estimated that in Paraguay 165,000 people are infected with Trypanosoma cruzi, the etiological agent of Chagas disease. Among them 61,000 are women of childbearing age. In 1995, it was implemented the Chagas Prenatal Control Program in the Cordillera department, one of the endemic areas of the country. Herein is analyzed the evolution of $T$. cruzi seropositivity in pregnant women in the 21 years of prenatal control (period: 1995-2016), with emphasis on the period 2010-2016. In this descriptive study, data from the registers of the III Sanitary Region and from the five diagnostic laboratories were used. The serologic results of 20 districts involved 23,661 pregnant women who attended their prenatal control. It was recorded 1,074 seropositive women in the five reference laboratories, resulting in a $5 \%$ prevalence of infection with $T$. cruzi in the period 2010-2016. At the beginning of the Program the prevalence was $16 \%$, the main decrease, to $6 \%$, was evidenced in 2014 maintaining quasi stationary until 2016. The Chagas disease prenatal diagnosis system and the protocols of prevention by the Ministry of Public Health and Social Welfare were effective. This information is useful to control the congenital transmission through the identification of positive pregnant women and providing the adequate treatment.

Keywords: Pregnant women, prenatal control, Chagas disease, seroprevalence.
\end{abstract}

\section{INTRODUCCION}

La enfermedad de Chagas, también llamada tripanosomiasis americana, es una enfermedad potencialmente mortal causada por el parásito protozoo Trypanosoma cruzi. Los principales vectores son insectos de la especie triatominos, siendo el principal vector en América del Sur el Triatoma infestans ${ }^{(1,2)}$. Según la OPS/OMS, esta enfermedad constituye uno de los principales problemas de salud pública en diversos países, principalmente en América Latina. Aproximadamente 6 millones de personas están infectadas por el $T$. cruzi, mientras que más de 70 millones de personas en las Américas viven en áreas de riesgos y actualmente se ha propagado a otros continentes ${ }^{(3)}$.

Clínicamente se reconocen 3 etapas de la enfermedad; la inicial o aguda que es de corta duración y está separada por una etapa asintomática indeterminada para luego entrar poco a poco en la etapa crónica la que generalmente aparece tardíamente. Las lesiones se producen principalmente en el miocardio y vísceras ${ }^{(4-6)}$ presentando los enfermos crónicos hasta un $30 \%$ alteraciones cardiacas y un $10 \%$ alteraciones digestivas, neurológicas o combinadas ${ }^{(6,7)}$.

La transmisión de la enfermedad de Chagas en humanos puede ocurrir por diversos mecanismos, la más importante es la vectorial que se realiza por la deyección de triatominos (vinchuca o chicha guazú), pero también están la transplacentaria que determina la infección congénita o vertical, por transfusiones sanguíneas lo que constituye un peligro real en zonas no endémicas, por trasplante de órganos infectados a receptores seronegativos, la transmisión por alimentos contaminados con heces de triatominos infectados, y los accidentes en laboratorios por manipulación de sangre $o$ animales infectados ${ }^{(7-10)}$.

Las mujeres en edad fértil que han adquirido la infección con el $T$. cruzi mayormente por transmisión vectorial en zonas endémicas son las que estando embarazadas transmiten la enfermedad de Chagas a sus hijos por vía transplacentaria, llamada también transmisión congénita o transmisión vertical. La transmisión congénita ocurre en todas las regiones endémicas de América Latina y se 
Meza Acosta G, Cerecetto Meyer H. Seroprevalencia de la enfermedad de Chagas en embarazadas...

estima que aproximadamente 15.000 infantes nacen infectados anualmente por transmisión vertical siendo el número de mujeres seropositivas de 15 a 44 años de alrededor de $1.809 .507^{(4,11)}$.

El diagnóstico de la enfermedad en fase aguda requiere de la detección de los parásitos, para lo cual se emplean diversas técnicas, entre ellas la parasitológica como observación directa al microscopio, moleculares como la reacción en cadena de la polimerasa (PCR), serológicas por técnicas ELISA, ELISA-SAPA e inmunofluorescencia $(\mathrm{IFI})^{(11-13)}$.

En la fase crónica de la enfermedad de Chagas los métodos parasitológicos para el diagnóstico poseen una sensibilidad menor, por lo que se recurre a métodos serológicos para la detección de anticuerpos, como la prueba de la técnica de ELISA. En este sentido, el reactivo utilizado por el Programa Nacional de Control de la Enfermedad de Chagas en Paraguay para el tamizaje en áreas endémicas y también para el control de la transmisión congénita es el Kit ELISA Chagas IICS v.1 de producción nacional que se viene utilizando en el país desde $1987^{(14)}$.

Según el Programa Nacional de Control de la Enfermedad de Chagas - SENEPA en Paraguay la vía más importante de transmisión de $T$. cruzi es la infección congénita o vertical; esto se debe probablemente al cumplimiento de la obligatoriedad del tamizaje serológico del $100 \%$ de los volúmenes de sangre a transfundir y a los avances significativos en el control vectorial, implementadas por más de una década manteniendo el logro del 2008, en cuanto a la interrupción de la transmisión vectorial de $T$. cruzi por el $T$. infestans en la Región Oriental ${ }^{(14,15)}$.

Los índices de infestación triatomínica en el Chaco Paraguayo registran un importante descenso, con niveles actuales de infestación promedios del $9 \%$, habiéndose registrado áreas de infestación del $50 \%$ en el año 2002, se ha logrado una cobertura del $90 \%$ del territorio chaqueño, sin embargo, aún se mantiene el riesgo de transmisión vectorial ${ }^{(15,16)}$.

En el año 2016, la Comisión Internacional de Evaluación concluyó que "en el departamento de Boquerón, Región Occidental del país, se ha logrado con más de 10 años de trabajo, indicadores entomológicos, seroepidemiológicos y clínicos que demuestran la interrupción de la transmisión vectorial domiciliaria de $T$. cruzi por $T$. infestans" ${ }^{\prime 16,17)}$.

La población expuesta en zona endémica es del $30 \%$ de la población total del país, con una estimación de 165.000 personas infectadas, una prevalencia país en bancos de sangre del $2,85 \%$ y una frecuencia de transmisión congénita del $5 \%{ }^{(14,17)}$.

En cuanto a la distribución de la enfermedad de Chagas en nuestro país, abarca todo el territorio nacional, con diferentes niveles de endemicidad; pudiendo presentarse casos de Chagas en personas procedentes o con antecedentes maternos de habitar departamentos de alta endemicidad (Concepción, Cordillera, Paraguarí, San Pedro y el Chaco), o en departamentos de mediana endemicidad (Guaira, Caaguazú, Misiones, Neembucú), siendo los de baja endemicidad Itapúa, Alto Paraná, Amambay, Canindeyú, Caazapá y Central ${ }^{(14,15)}$.

El departamento de Cordillera es el tercer departamento del país, es una zona endémica de la Región Oriental, donde la seroprevalencia de la enfermedad de Chagas en embarazadas del grupo etario de 15 a 45 años era del $15 \%$ en la década de los $90^{(11)}$. En julio de 1995 se estableció el Programa de Control Prenatal de Chagas en este departamento ${ }^{(18)}$.

En un estudio realizado en el 2016 con embarazadas del departamento de Cordillera, se encontró que la prevalencia de la infección con $T$. cruzi en 1997 fue del $15 \%$ y en el 2011 fue del $6 \%{ }^{(18)}$.

En el 2011, en el banco de sangre del departamento de Cordillera se detectó una prevalencia del 5,91 \%, siendo uno de los departamentos con frecuencia de casos reactivos mayor a la media nacional, la que se ubicaba en $2,92 \%{ }^{(17)}$.

En cuanto al Chagas congénito, la prevalencia de la trasmisión congénita en 12 años fue del 2 al $7 \%^{(11)}$. En estudios realizados a nivel nacional en niños de uno a 
cinco años, en el 2001 y el 2008, se registró que el departamento de Cordillera presentaba una mayor seropositividad, del $1,4 \%$ y $1,38 \%$, respectivamente ${ }^{(19)}$.

Por lo expuesto anteriormente y teniendo en cuenta que la enfermedad de Chagas en el país sigue siendo un problema de salud pública(10,13), se realizó este estudio sobre la seroprevalencia de la enfermedad de Chagas en embarazadas del departamento de Cordillera en el período 2010-2016, quince años después de la implementación del Programa de Control Prenatal de Chagas, y el comportamiento de la prevalencia de la enfermedad en embarazadas en los 21 años de control prenatal (período 1995-2016).

\section{MATERIAL Y MÉTODOS}

\section{Descripción de la zona de estudio}

Departamento de Cordillera de la región oriental, zona endémica del Paraguay (Figura 1). Limita al norte con San Pedro, al este con Caaguazú, al sur con Paraguarí y Central y al oeste con el rio Paraguay que lo separa de Presidente Hayes.

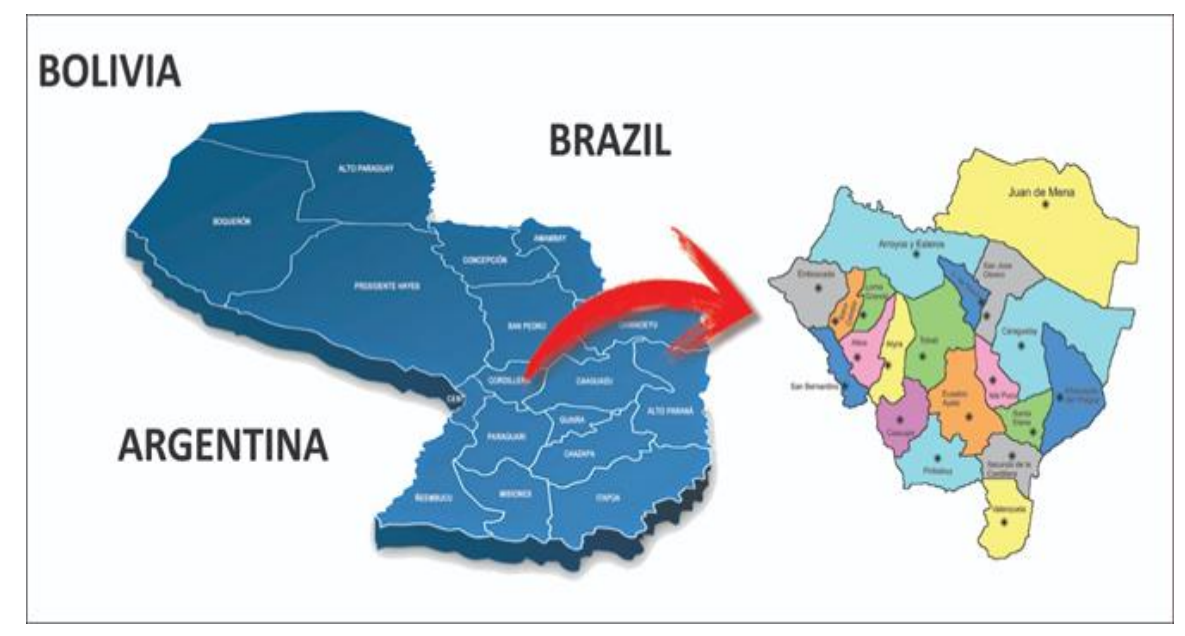

Figura 1: Zona de estudio. Mapa del Paraguay. Se señala la ubicación del Departamento de Cordillera con sus respectivas localidades donde se realizó el estudio.

Diseño del estudio. Encuestas seriadas de datos secundarios.

Área y población estudiada: Departamento de Cordillera de la Región Oriental, zona endémica del Paraguay para la enfermedad de Chagas. La población de estudio fueron las embarazadas que concurrieron a su control prenatal en los hospitales y centros de salud de los veinte distritos del departamento de Cordillera entre 2010 y 2016 . Se utilizaron los registros de las embarazadas que asistieron a su control prenatal y que fueron diagnosticadas como seropositivas para la enfermedad de Chagas en los cinco laboratorios de referencia del departamento de Cordillera.

Los cinco laboratorios de diagnóstico y los veinte distritos de los cuales se recibieron los datos fueron:

Laboratorio de diagnóstico de Caacupé: Altos, Atyra, Caacupé, Loma Grande, Mbocayaty del Yhaguy, Nueva Colombia, Piribebuy, San Bernardino.

Laboratorio de Arroyos Esteros: Arroyos y Esteros, Emboscada, Juan de Mena. 
Meza Acosta G, Cerecetto Meyer H. Seroprevalencia de la enfermedad de Chagas en embarazadas...

Laboratorio de Caraguatay: Caraguatay, Primero de Marzo, San José Obrero.

Laboratorio de Eusebio Ayala: Eusebio Ayala, Isla Pucú, Itacurubí de la Cordillera, Santa Elena, Valenzuela.

Laboratorio de Tobati: Tobatí.

Origen de los datos: A través de notas expedidas por la Facultad de Ciencias Exactas y Naturales de la Universidad Nacional de Asunción a la III Región Sanitaria del departamento de Cordillera, se accedió y se utilizaron los registros de embarazadas (fuente secundaria de información) dentro del Programa de Control Prenatal de Chagas del departamento de Cordillera desde 1995 hasta 2016. La población enfocada para conocer la seroprevalencia entre el 2010 y el 2016 fue un total de 23.661 embarazadas y para el estudio del comportamiento de la seroprevalencia en 21 años de implementación del dicho programa fue de 69.643 de embarazadas.

Método: El análisis se realizó sobre los registros secundarios disponibles en la III Región Sanitaria y en los cinco laboratorios de diagnóstico con los resultados del Test de ELISA para Chagas en embarazadas que concurrieron para su control prenatal entre 2010 y 2016. Con el análisis realizado se obtuvieron las frecuencias de embarazadas infectadas con T. cruzi en el periodo 2010-2016.

Técnicas de análisis: Los datos obtenidos a partir de los registros prenatales y las planillas resúmenes mensuales de los resultados de test de ELISAS para Chagas en embarazadas que concurrieron para su control prenatal entre 2010 y 2016 fueron agrupados en una base de datos, que luego fueron procesados y analizados por estadística descriptiva, a través del Programa Microsoft Office Excel 10, donde se analizó la distribución de los datos por medio de la confección de tablas y figuras, expresando los hallazgos en forma de frecuencia absoluta y porcentual.

\section{RESULTADOS}

Con el análisis de la base de datos, elaborada con los registros de las embarazadas que asistieron a su control prenatal a los hospitales y centros de salud de los 20 distritos del departamento de Cordillera entre los años 2010 y 2016, se pudo determinar la seroprevalencia de la enfermedad en ese período de tiempo.

Como se puede observar en la Tabla 1 en el período de estudio 7 años (2010-2016) se analizaron en los cinco laboratorios de diagnóstico un total de 23.661 muestras de embarazadas y se diagnosticaron 1.074 casos positivos, después de 15 años de implementación del Programa de Control Prenatal de Chagas (2010), siendo la prevalencia promedio de seropositividad en el departamento de Cordillera y en el período de estudio de 4,53\%. La prevalencia de la infección chagásica más baja registrada fue en los años 2013 y 2014. La prevalencia se mantuvo sin mayor variación (entre $3,55 \%$ y $6,32 \%$ ) en los años estudiados, pudiendo ser resultado de la efectividad del control implementado en el departamento de Cordillera. 
Tabla 1: Resultados de los análisis de la presencia de anticuerpos anti- $T$. cruzi realizados a mujeres embarazadas durante el control prenatal en el departamento de Cordillera entre los años 2010 y 2016.

\begin{tabular}{|c|c|c|c|c|c|c|c|c|c|c|c|c|c|c|c|c|c|}
\hline $\begin{array}{l}\text { Laboratorio de } \\
\text { diagnóstico }\end{array}$ & $\begin{array}{l}\text { AÑo } \\
\text { Distrito }\end{array}$ & Positivo & \begin{tabular}{|l|}
\multicolumn{2}{|c|}{2010} \\
Muestras \\
analizadas
\end{tabular} & Positivo & \begin{tabular}{l}
\multicolumn{2}{c|}{011} \\
Muestras \\
analizadas
\end{tabular} & Positivo & $\begin{array}{l}2012 \\
\begin{array}{l}\text { Muestras } \\
\text { analizadas }\end{array}\end{array}$ & Positivo & \begin{tabular}{l}
\multicolumn{2}{c}{2013} \\
Muestras \\
analizadas
\end{tabular} & Positivo & \begin{tabular}{l}
\multicolumn{1}{c|}{2014} \\
Muestras \\
analizadas
\end{tabular} & Positivo & $\begin{array}{l}2015 \\
\text { Muestras } \\
\text { analizadas }\end{array}$ & Positivo & \begin{tabular}{|l|}
2016 \\
Muestras \\
analizadas
\end{tabular} & $\begin{array}{l}\text { Total } \\
\text { Positivo }\end{array}$ & $\begin{array}{l}\text { Total } \\
\text { Muestras } \\
\text { analizadas }\end{array}$ \\
\hline \multirow[t]{8}{*}{ Caacupé } & Altos & 38 & 302 & 21 & 186 & 20 & 323 & 20 & 293 & 19 & 262 & 21 & 274 & 29 & 248 & 168 & 2056 \\
\hline & $\begin{array}{l}\text { Atyra } \\
\text { Caacupé }\end{array}$ & $\begin{array}{r}9 \\
56\end{array}$ & $\begin{array}{r}117 \\
1125\end{array}$ & $\begin{array}{r}6 \\
44\end{array}$ & $\begin{array}{l}112 \\
821\end{array}$ & $\begin{array}{l}12 \\
39\end{array}$ & $\begin{array}{l}170 \\
930\end{array}$ & $\begin{array}{l}12 \\
42\end{array}$ & $\begin{array}{r}204 \\
1051\end{array}$ & $\begin{array}{r}6 \\
32\end{array}$ & $\begin{array}{r}141 \\
1045\end{array}$ & $\begin{array}{l}11 \\
69\end{array}$ & $\begin{array}{r}170 \\
1086\end{array}$ & $\begin{array}{r}5 \\
69\end{array}$ & $\begin{array}{r}131 \\
1043\end{array}$ & $\begin{array}{r}61 \\
351\end{array}$ & $\begin{array}{l}1106 \\
7452\end{array}$ \\
\hline & Loma Grande & 1 & 30 & 5 & 28 & 3 & 35 & 0 & 30 & 0 & 18 & 2 & 51 & 2 & 46 & 13 & 251 \\
\hline & $\begin{array}{l}\text { Mbocayaty } \\
\text { del Yhaguy }\end{array}$ & 3 & 71 & 3 & 27 & 0 & 23 & 1 & 32 & 2 & 29 & 1 & 38 & 0 & 30 & 10 & 260 \\
\hline & $\begin{array}{l}\text { Nueva } \\
\text { Colombia }\end{array}$ & 3 & 32 & 5 & 50 & 2 & 42 & 2 & 28 & 3 & 36 & 3 & 50 & 5 & 33 & 23 & 294 \\
\hline & Piribebuy & 8 & 175 & 6 & 150 & 8 & 164 & 5 & 143 & 5 & 149 & 8 & 243 & 10 & 210 & 50 & 1284 \\
\hline & $\begin{array}{l}\text { San } \\
\text { Bernardino }\end{array}$ & 1 & 66 & 1 & 32 & 8 & 82 & 4 & 79 & 3 & 53 & 5 & 98 & 5 & 83 & 27 & 520 \\
\hline & Total & 119 & 1918 & 91 & 1406 & 92 & 1769 & 86 & 1860 & 70 & 1733 & 120 & 2010 & 125 & 1824 & 703 & 13223 \\
\hline \multirow[t]{4}{*}{$\begin{array}{l}\text { Arroyo y } \\
\text { Estero }\end{array}$} & $\begin{array}{l}\text { Arroyos y } \\
\text { Esteros }\end{array}$ & 17 & 425 & 29 & 346 & 11 & 463 & 5 & 326 & 9 & 301 & 17 & 388 & 9 & 438 & 97 & 2784 \\
\hline & Emboscada & 0 & 0 & 0 & 0 & 0 & 0 & 0 & 0 & 0 & 0 & 0 & 0 & 0 & 0 & 0 & 0 \\
\hline & $\begin{array}{l}\text { Juan de } \\
\text { Mena }\end{array}$ & 5 & 65 & 5 & 90 & 5 & 74 & 3 & 76 & 4 & 76 & 1 & 108 & 2 & 115 & 25 & 629 \\
\hline & Total & 22 & 490 & 34 & 436 & 16 & 537 & 8 & 402 & 13 & 377 & 18 & 496 & 11 & 553 & 122 & 3413 \\
\hline \multirow[t]{3}{*}{ Caraguatay } & $\begin{array}{l}\text { Caraguatay } \\
\text { Primero de } \\
\text { Marzo }\end{array}$ & $\begin{array}{l}3 \\
\mathbf{0}\end{array}$ & $\begin{array}{r}118 \\
31\end{array}$ & $\begin{array}{l}3 \\
1\end{array}$ & $\begin{array}{r}105 \\
29\end{array}$ & $\begin{array}{l}1 \\
1\end{array}$ & $\begin{array}{l}55 \\
18\end{array}$ & $\begin{array}{l}3 \\
1\end{array}$ & $\begin{array}{r}114 \\
54\end{array}$ & $\begin{array}{l}\mathbf{1} \\
\mathbf{0}\end{array}$ & $\begin{array}{l}78 \\
14\end{array}$ & $\begin{array}{l}1 \\
2\end{array}$ & $\begin{array}{l}77 \\
23\end{array}$ & $\begin{array}{l}9 \\
2\end{array}$ & $\begin{array}{r}124 \\
42\end{array}$ & $\begin{array}{r}21 \\
7\end{array}$ & $\begin{array}{l}692 \\
218\end{array}$ \\
\hline & $\begin{array}{l}\text { San José } \\
\text { Obrero }\end{array}$ & 3 & 20 & 0 & 14 & 0 & 1 & 0 & 17 & 0 & 8 & 0 & 2 & 0 & 9 & 3 & 74 \\
\hline & Total & 6 & 169 & 4 & 148 & 2 & 74 & 4 & 185 & 1 & 100 & 3 & 102 & 11 & 175 & 31 & 984 \\
\hline \multirow[t]{5}{*}{$\begin{array}{l}\text { Eusebio } \\
\text { Ayala }\end{array}$} & $\begin{array}{l}\text { Eusebio } \\
\text { Ayala }\end{array}$ & 12 & 356 & 12 & 206 & 7 & 58 & 4 & 49 & 0 & 70 & 9 & 209 & 17 & 256 & 61 & 1265 \\
\hline & Isla Pucu & 3 & 23 & 0 & 12 & 0 & 4 & 0 & 0 & 0 & 0 & 0 & 0 & 0 & 0 & 3 & 42 \\
\hline & $\begin{array}{l}\text { Itacurubi de } \\
\text { la Cordillera }\end{array}$ & 2 & 71 & 4 & 62 & 2 & 35 & 0 & 26 & 0 & 68 & 1 & 80 & 3 & 78 & 12 & 432 \\
\hline & $\begin{array}{l}\text { Santa Elena } \\
\text { Valenzuela }\end{array}$ & $\begin{array}{l}1 \\
2\end{array}$ & $\begin{array}{l}41 \\
41\end{array}$ & $\begin{array}{l}\mathbf{0} \\
\mathbf{0}\end{array}$ & $\begin{array}{l}12 \\
11\end{array}$ & $\begin{array}{l}\mathbf{0} \\
\mathbf{0}\end{array}$ & $\begin{array}{l}12 \\
33\end{array}$ & $\begin{array}{l}1 \\
0\end{array}$ & $\begin{array}{l}13 \\
35\end{array}$ & $\begin{array}{l}1 \\
3\end{array}$ & $\begin{array}{l}33 \\
41\end{array}$ & $\begin{array}{l}2 \\
4\end{array}$ & $\begin{array}{l}46 \\
60\end{array}$ & $\begin{array}{l}2 \\
4\end{array}$ & $\begin{array}{l}60 \\
51\end{array}$ & $\begin{array}{r}7 \\
13\end{array}$ & $\begin{array}{l}224 \\
285\end{array}$ \\
\hline & Total & 20 & 532 & 16 & 303 & 9 & 142 & 5 & 123 & 4 & 212 & 16 & 395 & 26 & 445 & 96 & 2248 \\
\hline \multirow[t]{2}{*}{ Tobati } & Tobati & 24 & 337 & 23 & 362 & 20 & 408 & 11 & 427 & 12 & 290 & 19 & 459 & 13 & 314 & 122 & 2719 \\
\hline & Total & 24 & 337 & 23 & 362 & 20 & 408 & 11 & 427 & 12 & 290 & 19 & 459 & 13 & 314 & 122 & 2719 \\
\hline \multirow[t]{3}{*}{ Total } & & 191 & 3446 & 168 & 2655 & 139 & 2930 & 114 & 2997 & 100 & 2712 & 176 & 3462 & 186 & 3311 & 1074 & 23661 \\
\hline & & $5,54 \%$ & & $6,32 \%$ & & $4,74 \%$ & & $3,80 \%$ & & $3,55 \%$ & & $5 \%$ & & $5,6 \%$ & & $4,53 \%$ & \\
\hline & $\begin{array}{l}\text { Prevalencia } \\
\text { Años }\end{array}$ & $6 \%$ & 2010 & $6 \%$ & 2011 & $5 \%$ & 2012 & $4 \%$ & 2013 & $4 \%$ & 2014 & $5 \%$ & 2015 & $6 \%$ & 2016 & $5 \%$ & \\
\hline
\end{tabular}

Fuente: Base de datos proveído por la III Región Sanitaria de Cordillera. Año 2018 
Por otro lado, el total de muestras analizadas en 21 años (1995 a 2016) de implementación del Programa de Control Prenatal de Chagas en embarazadas del departamento de Cordillera fue de 69.693 diagnosticándose 6.651 casos positivos.

Al inicio de la implementación del Programa de Control Prenatal de Chagas la prevalencia era de $16 \%$ (Figura 2) y la misma fue descendiendo paulatinamente hasta el 2004 en que se alcanzó una prevalencia de $6 \%$, manteniéndose luego en una fase cuasi estacionaria con una prevalencia promedio de $6 \%$ entre 2005 y 2016, siendo el descenso de la prevalencia de 10 unidades desde 1995 a 2016, con un porcentaje de disminución del $62,5 \%$. La prevalencia promedio más baja se detectó en 2013 y 2014 con un valor de $4 \%$.

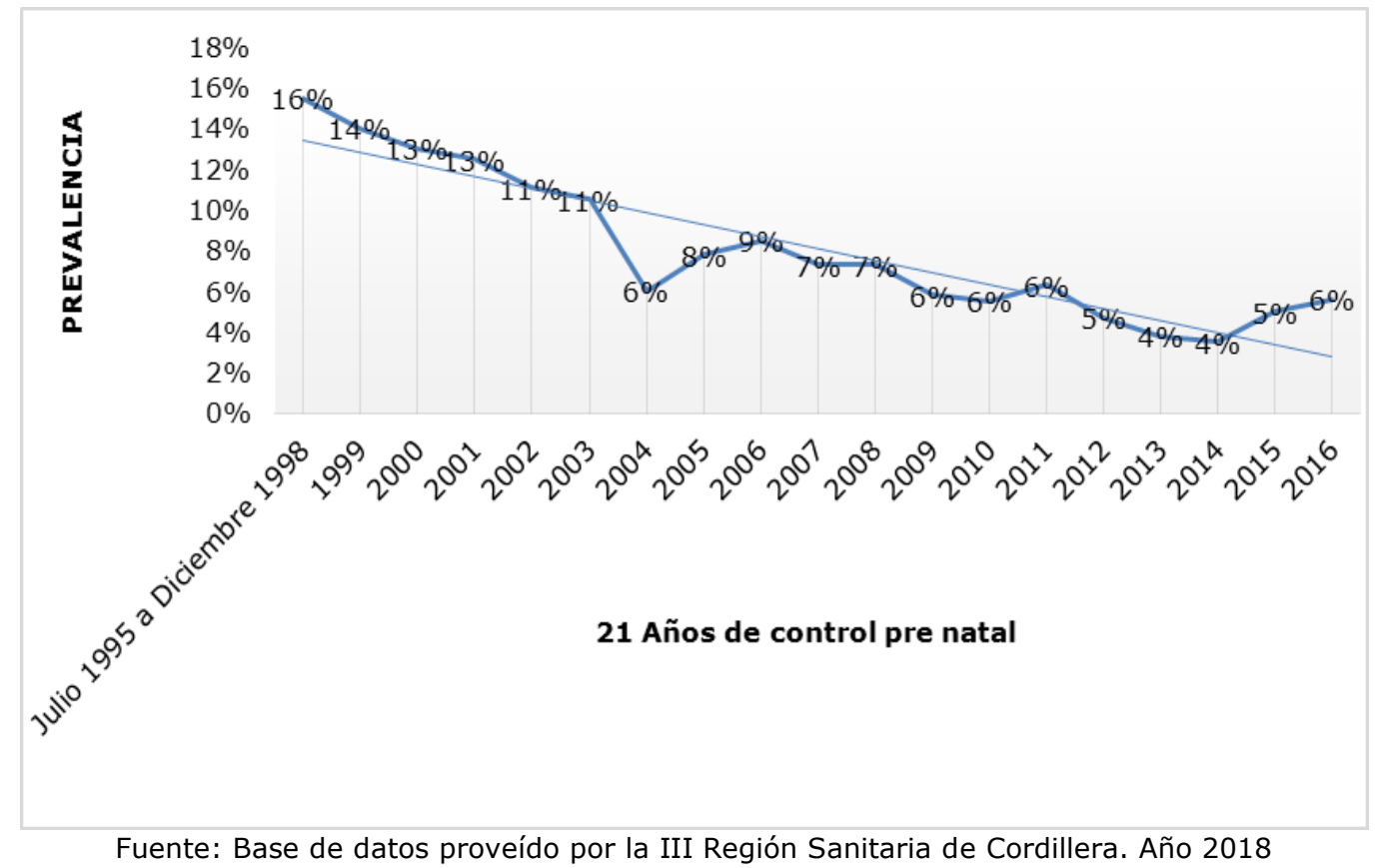

Figura 2: Prevalencia de seropositividad para $T$. cruzi en mujeres embarazadas analizadas durante el control prenatal en el departamento de Cordillera entre 1995 y 2016.

Continuando con la investigación de nuestro estudio previo de la seroplevalencia de la enfermedad de Chagas en embarazadas en los mismos distritos del departamento de Cordillera (Tabla 2), se evidenció que los valores resultaron siempre menores a los registrados en el año 1997 con una disminución de la seropositividad, promedialmente, del 69,2 \%, desde 1997 al 2016, siendo Caraguatay el distrito con la máxima disminución promedial de la seropositividad, 82 \% y el distrito de Eusebio Ayala con la mínima disminución promedial, $48 \%$. Los distritos con las mayores seropositividades en el año 1997 fueron Altos, Nueva Colombia, San Bernardino y Loma Grande, han conseguido disminuciones significativas de estos valores, en el entorno del $70 \%$ en el período 2011-2016, pero continúan siendo unos de los distritos con mayor porcentaje de seropositividad en el período 2011-2016, con valores promedio de 8,$4 ; 8,6 ; 5,8$ y $5,8 \%$, respectivamente. 
Tabla 2: Seroprevalencia de la enfermedad de Chagas por distrito del departamento de Cordillera en los años 1997 y 2011-2016.

\begin{tabular}{|c|c|c|c|c|c|c|c|c|c|c|c|c|c|c|c|}
\hline \multirow[b]{2}{*}{ Año/Distrito } & \multicolumn{15}{|c|}{ Seroprevalencia promedio (\%) } \\
\hline & A & NC & SB & LG & $\mathbf{P}$ & C & $\mathbf{T}$ & JM & 1M & At & $\mathbf{C a}$ & $\mathbf{M}$ & EA & $\mathbf{A E}$ & IC \\
\hline 1997 & 29 & 26 & 25 & 25 & 18 & 16 & 15 & 15 & 14 & 13 & 12 & 12 & 12 & 11 & 8 \\
\hline 2011 & 11 & 10 & 3 & 18 & 4 & 3 & 6 & 6 & 3 & 5 & 5 & 11 & 6 & 8 & 6 \\
\hline 2012 & 6,2 & 4,8 & 9,8 & 8,6 & 4,9 & 1,8 & 4,9 & 3,0 & 5,8 & 7,1 & 4,2 & 0,0 & 12,1 & 2,4 & 5,7 \\
\hline 2013 & 6,8 & 7,1 & 5,1 & 0,0 & 3,5 & 2,6 & 2,6 & 2,0 & 1,8 & 5,9 & 4,0 & 3,1 & 8,2 & 1,5 & 0,0 \\
\hline 2014 & 7,3 & 8,3 & 5,7 & 0,0 & 3,3 & 1,3 & 4,1 & 3,4 & 0,0 & 4,2 & 3,1 & 6,7 & 0,0 & 3,0 & 0,0 \\
\hline 2015 & 7,7 & 6,0 & 5,1 & 3,9 & 3,3 & 1,3 & 4,1 & 3,6 & 8,7 & 6,5 & 6,3 & 2,6 & 4,3 & 4,4 & 1,2 \\
\hline 2016 & 11,7 & 15,2 & 6,0 & 4,3 & 3,8 & 7,3 & 4,1 & 1,7 & 4,8 & 3,8 & 6,6 & 0,0 & 6,6 & 2,0 & 3,8 \\
\hline $\begin{array}{l}\text { Promedio 2011- } \\
2016\end{array}$ & 8,4 & 8,6 & 5,8 & 5,8 & 3,8 & 2,9 & 4,3 & 3,3 & 4,0 & 5,4 & 4,9 & 3,9 & 6,2 & 3,5 & 2,8 \\
\hline $\begin{array}{l}\text { Disminución } \\
\text { 1997-2016 (\%) }\end{array}$ & 71 & 67 & 77 & 77 & 79 & 82 & 71 & 78 & 71 & 58 & 59 & 67 & 48 & 68 & 65 \\
\hline
\end{tabular}

\section{DISCUSIÓN}

En este estudio se analizó la seroprevalencia de la enfermedad de Chagas en embarazadas que asistieron a su control prenatal en los hospitales y centros de salud de los 20 distritos que componen el departamento de Cordillera, después de 21 años de la implementación del Programa de Control Prenatal de la enfermedad de Chagas. Se encontró que, en el período analizado (2010-2016), se mantuvieron los valores de seroprevalencia en el entorno del $5 \%$. Resultando, desde la implementación del programa, un descenso de la prevalencia, de 10 unidades, con un porcentaje de disminución del $62,5 \%$. Nuestro estudio previo en la población de embarazadas de la misma región ${ }^{(18)}$ encontró una disminución de la seroprevalencia, analizando resultados de 1997 y 2011, similar a la descrita aquí.

En cuanto al comportamiento de la prevalencia de la enfermedad de Chagas en embarazadas en 21 años de control prenatal en el departamento de Cordillera, nueve años después de la implementación en 2004, se observó el máximo descenso, llegando a una prevalencia de $6 \%$, situación que fue seguida con una fase cuasi estacionaria con un valor promedio de $6 \%$ entre 2005 y 2016 resultando una prevalencia de seropositividad promedio en el departamento de Cordillera de $5 \%$. Russomando describe en el 2009 que entre 2005 y 2009 hubo un descenso importante de la prevalencia de la enfermedad de Chagas en embarazadas del grupo etario de 15 a 45 años, con valores del $8 \%$ a $6 \%$, como resultado de las acciones de control vectorial iniciadas en 1999 en forma continua en el tiempo y al tamizaje serológico en bancos de sangre que alcanzó el $100 \%{ }^{(11)}$.

Continuando con nuestro estudio previo(18), se analizó la evolución en el descenso, durante el período 2011-2016, de la seroprevalencia de la enfermedad de Chagas en las embarazadas, por distritos del departamento de Cordillera. Así, fue posible evidenciar que en todos los distritos analizados del departamento de Cordillera los valores resultaron siempre menores que los registrados en el año 1997 con una disminución de la seropositividad, promedialmente, del 69,2 \%, desde 1997 al 2016, siendo Caraguatay el distrito con la máxima disminución promedial de la seropositividad, $82 \%$, y el distrito de Eusebio Ayala con la mínima disminución, promedial, 48\%. Cabe destacar que los distritos con las mayores seropositividades en el año 1997, a saber, Altos, Nueva Colombia, San Bernardino y Loma Grande, han conseguido disminuciones significativas de estos valores, en el entorno del $70 \%$ en el período 2011-2016, pero continúan siendo unos de los distritos con mayor porcentaje de seropositividad en el período 2011-2016, con valores promedio de 8,4; 8,6; 5,8 y $5,8 \%$, respectivamente. La ubicación geográfica de estos distritos en el departamento de Cordillera, hacia el oeste del mismo, podría ser uno de los motivos a considerar en 
estudios posteriores, donde las gestiones de los procedimientos de control de la enfermedad podrían no ser lo efectivas que deberían ser.

En este estudio se pudo observar que la prevalencia desde el año 2010 al 2016 se mantiene en un promedio del $5 \%$ que puede ser atribuida a infecciones distintas a la vectorial; así mismo en la publicación del SENEPA 2017 se menciona que la prevalencia en la región oriental es hasta el $5 \%$, considerando que la vía más importante de transmisión es la vertical; este fenómeno se debe al tamizaje serológico del $100 \%$ de los volúmenes de sangre a transfundir y a los avances en control vectorial re-certificados por el INCOSUR ${ }^{(20)}$.

La prevalencia encontrada en el departamento de Cordillera, zona endémica del país a inicio de la implementación del programa de control de la enfermedad de Chagas en embarazadas fue del $16 \%$. Se constató una tendencia general de disminución de $6 \%$, un estudio reciente realizado en la República de Argentina según riesgo de transmisión vectorial e indicadores de controles en embarazadas infectadas y sus hijos, en 2002 y 2014 demostró un comportamiento similar en la provincia Chaco que es una zona de alta endemicidad en la cual en el año 2002 la prevalencia de infección en embarazadas fue de 15.9 y en el 2014 fue del $10.4 \%{ }^{(22)}$.

En este estudio se estima que, en el departamento de Cordillera, la III Región Sanitaria ha evaluado serológicamente más del $90 \%$ de las mujeres en edad fértil, en un estudio previo del 2009 Russomando estimó que la cobertura fue del $70 \%{ }^{(11)}$.

Según la Dirección General de Estadística Encuestas y Censo el porcentaje de la población de mujeres en edad fértil presentara un aumento del $51 \%$ en el año 2019 a $52 \%$ para el año $2024^{(23,24)}$, es decir, más de la mitad de la población femenina tendría entre 15 a 49 años de edad, teniendo en cuenta que la prevalencia de la trasmisión congénita en 12 años fue del 2 al $7 \%^{(11)}$ lo que evidencia que sigue siendo un problema de salud pública. Por lo que el sistema de control prenatal de la enfermedad de Chagas se debería fortalecer para mejorar la captación de los niños infectados para su tratamiento oportuno, teniendo en cuenta que los diferentes laboratorios de diagnósticos poseen registros fehacientes de las embarazadas seropositivas.

Una vigilancia instalada, sostenible y factible adaptada a las necesidades epidemiológicas de la zona sirve de guía para el buen funcionamiento del Programa de Control de Chagas en la eliminación de vectores de $T$. cruzi y en la prevención de la enfermedad ya que los registros ayudan en la toma de decisiones para las acciones efectivas en casos de infecciones verticales ${ }^{(15,21)}$. Los resultados obtenidos en este estudio indican que aún la seroprevalencia de la enfermedad en el departamento de Cordillera es considerable y que los trabajos de prevención de la transmisión congénita deben ser fortalecidos con sólidas estrategias de diagnóstico precoz y tratamiento oportuno en recién nacidos y niños infectados.

\section{AGRADECIMIENTOS}

A la III Región Sanitaria del departamento de Cordillera por los datos proveídos para la realización del trabajo de tesis, presentado en la Facultad de Ciencias Exactas y Naturales, Universidad Nacional de Asunción, para la obtención del título de Magíster en Elaboración, Gestión y Evaluación de Proyectos de Investigación Científica.

Financiación: Este trabajo fue financiado con fondos propios de los autores.

Conflicto de interés: Los autores declaran no tener ningún conflicto de interés.

\section{REFERENCIAS BIBLIOGRAFICAS}

1. WHO. First WHO report on neglected tropical diseases: Working to overcome the global impact of neglected tropical diseases /Internet/.Geneva: WHO; 2010 Disponible en:

http://www.who.int/neglected_diseases /2010report/en/\#.
2. Colombia. MINSALUD. Instituto Nacional de Salud. Protocolo de Vigilancia en Salud Pública. Chagas. Bogotá: MINSALUD; 2014.

3. OPS/OMS. "22a Reunión de la Comisión Intergubernamental del Cono Sur para la prevención y atención de la 
enfermedad de Chagas (INCOSUR/CHAGAS)". Certifican logro histórico de Paraguay en la lucha contra el Chagas. Asunción 23 nov.2016. Disponible:

www.mspbs.gov.py/certifican-logrohistorico-de-paraguay-en-la-luchacontra-el-chag.

4. Organización Panamericana de la Salud. Estimación cuantitativa de la enfermedad de Chagas en las Américas. Montevideo: OMS; 2006.

5. Brener Z, Andrade Z, Barral-Neto $M$. Trypanosoma cruzi e Doenca de Chagas. 2a edición. Rio de Janeiro: Editorial Guanabara Koogan. 2000.

6. Chagas Disease/Tripanosomiasis Americana Enfermedad de Chagas Chagas-Mazza factsheets [internet]. Iowa (College of Veterinary Medicine): Iowa State University; 2009. Disponible www.cfsph.iastate.edu/Factsheets/es/tr ypanosomiasis_american-es.pdf.

7. Organización Mundial de la Salud. La enfermedad de Chagas (tripanosomiasis americana). Nota descripta No 340: OMS; 2010.

8. Organización Mundial de la Salud. Control de la Enfermedad de Chagas. Segundo informe del Comité de Expertos de la OMS. Ginebra: OMS; 2002

9. Storino RAo, Milei J. Enfermedad de Chagas.Buenos Aires:Mosby, Doyma; 1994

10. Organización Panamericana de la Salud. XIa. Reunión de INCOSUR / Chagas, Asunción, Paraguay, marzo de 2002.

11. Russomando G. Transmisión congénita de la enfermedad de Chagas en el Paraguay. Mem. Inst. Cienc. Salud. 2009; 7(2):55-64.

12. Russomando G, Sanchez Z, Meza G, Guillen I. Shed acute-phase antigen protein in an ELISA system for unequivocal diagnosis of congenital Chagas disease. Expert Rev. Mol. Diagn. 2010; 10 (6), 705-7.

13. Russomando $G$, Almirón $M$, Candia $N$, Franco L, Sánchez $Z$, Guillen I. Implementación y evaluación de un sistema localmente sustentable de diagnóstico prenatal que permite detectar casos de transmisión congénita de la enfermedad de Chagas en zonas endémicas del Paraguay. Soc. Bras Med Trop 2005; 38(2): 49-54.

14. Programa del SENEPA: Chagas. 2013. programassenepa.blogspot.com/p/chag as.html

15. Rojas de Arias A. La certificación del corte de transmisión vectorial del Trypanosoma cruzi, agente etiológico de la enfermedad de Chagas. Mem. Inst.
Investig. Cienc. Salud. 2016; 14(3): 36.

16. OPS/OMS. Enfermedades infecciosas desatendidas en las Américas: Historias de éxito e innovación para llegar a los más necesitados. Washington, DC. 2016; Disponible:

https://www.paho.org/hq/index.php?op tion=com_docman\&task=doc_download \&Itemid=270\&gid=39112\&lang=es.

17. Marquez Roa NA, Lemir de Zelada MO, Molas AC. Frecuencia serológica de infección por Trypanosoma cruzi en donantes de sangre en el Paraguay entre los años 2006 y 2011. Mem. Inst. Investig. Cienc. Salud, 2013; 11(2): 2631.

18. Meza G. Seroprevalencia de la enfermedad de Chagas en embarazadas del departamento de Cordillera antes y después de la implementación del control prenatal de Chagas en los periodos 1997 y 2011. Mem. Inst. Investig. Cienc. Salud. 2016; 14(3):7380, doi: 10.18004/Mem.iics/18129528/2016.014(03)73-080

19. Russomando G, Cousiño B, Sánchez Z, Franco L, Nara EM, Chena L, et al. Chagas disease: national survey of seroprevalence in children under five years of age conducted in 2008. Mem. Inst. Oswaldo Cruz [Internet]. 2017 May [cited 2019 ago 29] ; 112( 5 ) : 348-53. Available from: http://www.scielo.br/scielo.php?script= sci_arttext\&pid=S007402762017000500348\&lng=en

20. SENEPA. Memoria Annual Institucional 2017.

21. Danesi E, Olenka Codebó M, Sosa S. Transmisión Congénita de Trypanosoma Cruzi. Argentina 2002-2014. MEDICINA (Buenos Aires) 2019; 79: 81-9.

22. Paraguay. Dirección General de Estadísticas Encuestas y Censo. Cordillera Proyección por sexo y edad. 2018 [internet].Asunción:dgeec;2018. Disponible:

https://www.dgeec.gov.py/.../Proyeccio nes\%20departamentos/03_Cordillera_C alculo_...

23. Paraguay. Dirección General de Estadísticas Encuestas y Censo. Cordillera Proyección por sexo y edad. 2019 [internet. ]Asunción:dgeec;2018. Disponible:

https://www.dgeec.gov.py/.../Proyecion es\%20por\%20Departamento\%202019/ 03_Cor...

24. DGEEC. Cordillera Proyección por sexo y edad. 1 mar. 2019. Disponible: https://www.dgeec.gov.py/Publicacione s/Proyeciones\%20por\%20Departament o\%202019/03_Cordillera_2019.pdf 